\title{
Saluto del Presidente APLOTO
}

\author{
Antonio Aloisi \\ Presidente APLOTO, Presidio Ospedaliero Galatina, Lecce, Italia \\ antonioaloisi@tin.it \\ Pubblicato online: 5 aprile 2017 \\ (c) Società Italiana Ortopedici Traumatologi Ospedalieri d'Italia 2017
}

Con grande piacere porto il saluto della nostra associazione nella presentazione di questo fascicolo del "Lo Scalpello" realizzato con il contributo di tanti ortopedici pugliesi e lucani capaci ed esperti e ringrazio l'OTODI per aver dato la possibilità di curarlo al nostro Chief Editor dottor Enzo Caiaffa e ai suoi collaboratori dottor Claudio Mori e dottor Nicola Tartaglia.

La Fissazione Esterna (FE) come trattamento di fratture complesse è un argomento interessante che, soprattutto in Puglia, ha trovato da sempre cultori ed esperti, stimati oltre i confini regionali; per questo basta ricordare il Secondo Corso Nazionale d'istruzione sulla fissazione esterna in Italia, tenutosi a Bari il 30 e 31 gennaio 1998 e presieduto dal Prof. G.B. Solarino, per capire quanto il nostro cammino sia lungo in questo campo.

Da allora molte cose sono cambiate. Infatti, dopo qualche decennio di diffusione intensiva di tale tecnica, la presenza di sequele iatrogene prima sconosciute ha imposto una profonda riflessione sulle indicazioni. Molta acqua, a dire il vero, era già passata sotto i ponti dal 1853 , anno ufficiale di nascita della FE con il ragno patellare di Jean-François Malgaigne, la ganascia bivalve di Albin Lambotte nel 1902, il fissatore modulare di Raoul Hoffmann del 1938, il quadrato semplice di John C. Charnley del 1948, il circolare del siberiano Ilizarov degli anni '60 (si contano 150 modelli nel mondo) fino al fissatore di De Bastiani che nei primi anni '80 affermava "un metodo valido nei casi gravi è tanto più valido in quelli semplici", ribadendo l'ampia versatilità di questa tecnica, non solo utilizzabile ai fini del damage control (massima rapidità esecutiva, minima perdita ematica, minima emissione catabolica) ma anche come scelta definitiva per portare a guarigione lesioni scheletriche con punteggio di Injury Severity Score (ISS) inferiore anche a 16.

Oggi il miglioramento dei materiali e il perfezionamento delle tecniche chirurgiche ci consentono di affermare che la FE è metodica raccomandabile nel trattamento di gravi fratture esposte degli arti e del bacino e in pazienti politraumatizzati tanto da ritenerla, di fatto, una metodica salvavita, dando però contemporaneamente all'ortopedico la possibilità di eseguire eventuali correzioni al trattamento.

Questo fascicolo si pone dunque l'obiettivo di accrescere la formazione di tutti gli ortopedici e, in particolare, dei più giovani con un approfondimento a più voci che sicuramente chiarisce molte idee e può stimolare un positivo dibattito scientifico anche per uniformare alcuni trattamenti, troppo spesso personalizzati e incerti.

Inoltre, tale incontro di esperienze così mirabilmente rappresentate da tanti ottimi colleghi permette certamente di confrontare la FE con altri moderni mezzi di sintesi, definendone il proprio campo d'azione e migliorandola sul piano scientifico, tecnologico e nelle indicazioni chirurgiche.

Buona lettura e ad maiora semper! 